\title{
Single blastocyst transfer yields similar pregnancy rates compared with multiple cleavage embryo transfer, with reduced twin rate, in patients with low number of fertilized oocytes
}

\author{
Stephanie Smeltzer ${ }^{1 *}$, Kelly Acharya ${ }^{1}$, Tracy Truong ${ }^{2}$, Carl Pieper ${ }^{2}$ and Suheil Muasher ${ }^{1}$
}

\begin{abstract}
Background: In patients with low numbers of embryos, there is not yet consensus on whether to extend culture to the blastocyst stage, especially due to the risk that some or all of the embryos will not make it to the blastocyst stage. The objective of our study was to evaluate pregnancy outcomes in patients with a low number of fertilized oocytes $(<4)$, comparing single blastocyst transfer to one or more cleavage embryo transfer.

Results: We analyzed 6795 cycles from the 2014-2105 Society for Assisted Reproductive Technology (SART) registry. All patients were $\leq 38$ years old, had less than four fertilized oocytes, and were undergoing first fresh in vitro fertilization (IVF) transfer. Primary outcomes were clinical pregnancy $(C P)$, live birth $(L B)$, and miscarriage rate in both cleavage stage transfer and single blastocyst transfer. A secondary outcome was the rate of twin gestation. The comparison of interest in day of transfer included (1) single blastocyst vs single cleavage and (2) single blastocyst vs multiple cleavage stage. The association between day of transfer and primary outcome was investigated using logistic regression, controlling for the age, race/ethnicity, BMI, smoking, gravidity, parity, infertility diagnoses, and assisted hatching.

Single blastocyst transfer was associated with an increased odds of CP (adjusted OR 2.03) and LB (adjusted OR 1.86) when compared to single cleavage transfer, and no statistically significant association was observed when comparing single blastocyst transfer to multiple cleavage embryo transfer for CP (adjusted OR 0.94) and LB (adjusted $O R=0.88$ ). The odds of having twins among single blastocyst transfer was significantly lower compared to those odds that among multiple cleavage stage transfer (unadjusted OR 0.09).

Conclusions: While pregnancy outcomes are similar between single blastocyst and multiple cleavage embryo transfer, the twin rate is reduced significantly among the single blastocyst transfers in patients with a low number of fertilized oocytes.
\end{abstract}

Keywords: In vitro fertilization, Live birth, Clinical pregnancy, Poor responder

\footnotetext{
* Correspondence: stephanielsmeltzer@gmail.com

${ }^{1}$ Duke Fertility Center, Duke University, 5704 Fayetteville Road, Durham, NC

27713, USA

Full list of author information is available at the end of the article
} 


\section{Background}

In the current era of in vitro fertilization (IVF), many practitioners choose to extend embryo culture to the blastocyst stage as blastocyst transfers have shown an association of an improved clinical pregnancy $(\mathrm{CP})$ rate and live birth (LB) rate [1]. In a 2016 Cochrane review of 27 randomized clinical trials, the $\mathrm{LB}$ and $\mathrm{CP}$ rates were significantly higher in the blastocyst versus the cleavage group after a fresh transfer [2]. In a randomized control trial by Papanikolaou et al. in patients under age 36 , higher clinical pregnancy and live birth rates were seen with a single blastocyst compared to a single cleavage stage transfer [3].

Blastocyst transfer is also associated with other additional benefits. Foremost, with blastocyst transfer, there is a decreased risk of multiple gestation as less embryos can be transferred with a similar live birth rate. Blastocyst transfer is also more physiologic, as this is the stage when the embryo normally enters the uterus. Finally, blastocyst transfer allows for better selection of a viable embryo by morphology and also allows for the possibility of preimplantation genetic testing through embryo biopsy. As such, a single blastocyst transfer is now the preferred method of transfer, as it leads to improved pregnancy rates and a reduced risk of multiple gestation.

In extending culture to blastocyst stage, there is a risk that some or all of the embryos will not make it to blastocyst stage. In a study of 142 patients that received extended culture to the blastocyst stage, $22.5 \%$ of the embryos did not survive the extended culture [4]. In a meta-analysis by Papanikolaou et al., clinical pregnancy and live birth rates were higher in patients after a blastocyst transfer; however, these patients also had a higher cancelation rate [5]. In a prospective randomized study by Levitas et al., blastocyst transfer had a higher implantation rate in patients who failed to conceive in 2 or more day $2 / 3$ transfers, though a higher cancelation rate was seen [6]. It is of interest that in this study, the cancelation rate was reduced if the decision to transfer was made on day 3, a post hoc conclusion.

In patients with low numbers of embryos, there is not yet consensus on whether to extend these embryos to blastocyst. The objective of our study was to evaluate pregnancy outcomes in patients with a low number of fertilized oocytes $(<4)$, comparing single blastocyst transfer to one or more cleavage embryo transfer.

\section{Methods}

All autologous first fresh IVF cycles from the 2014-2015 Society for Assisted Reproductive Technology (SART) database were analyzed. We included cycles that were a first fresh IVF cleavage or blastocyst stage transfer and if age was less than or equal to 38. Cleavage stage transfer included cycles with one or more embryos transferred, and blastocyst transfer only included single embryo transfer. Cycles that had less than four fertilized oocytes and that used conventional IVF and intracytoplasmic sperm injection were included while donor oocyte cycles, gestational carrier cycles, any cycles that used PGT$\mathrm{A}$, and cycles that involved use of a combination of fresh embryos with cryopreserved embryos were excluded.

Demographic information included patient's age, race/ ethnicity, BMI (defined as weight in kilograms divided by height in meters squared), follicle stimulating hormone dose, gravity, parity, smoking status, and infertility diagnosis. In addition, cycle characteristics including the number of oocytes retrieved, the number of fertilized oocytes, the number of good quality embryos cryopreserved, and whether assisted hatching was performed are listed.

The IVF and pregnancy outcomes include CP rate (defined by in The International Glossary on Infertility and Fertility Care as the presence of ultrasonographic visualization of one or more gestational sacs or definitive signs of pregnancy), miscarriage rate (defined at the spontaneous loss of a CP before 22 weeks gestational age in which the embryo or fetus is nonviable), live birth (LB) rate (defined as the delivery from a women after 22 weeks completed gestational age), singleton rate (the rate of singleton pregnancies among all cycles with a heartbeat), and twin rate (the rate of twin pregnancies among all cycles with a heartbeat) [7]. All IVF and pregnancy outcomes refer to the first fresh embryo transfer outcome.

Statistical analysis was performed using R version 3.5.3 [8]. Demographic and patient characteristics were summarized using mean with standard deviation (SD), median with interquartile range (IQR), or frequency with percentage by day of transfer (single blastocyst, single cleavage, or multiple cleavage). The comparison groups of interest included (1) single blastocyst transfer vs single cleavage transfer and (2) single blastocyst transfer vs multiple cleavage transfer. Student's $t$ test and Wilcoxon rank sum test were used for continuous variables for between groups listed in (1) and (2) above, while Chi-square Goodness-of-Fit test was used between group comparisons of categorical variables. We investigated the association between day of transfer and each primary outcome (CP, LB, and miscarriage) using logistic regression, controlling for age, race/ethnicity, BMI, smoker, gravidity, parity (nulliparous and multiparous), infertility diagnoses (male factor, endometriosis, polycystic ovarian syndrome, diminished ovarian reserve, tubal factor, uterine factor, unexplained, and other reason), and assisted hatching. E-value was calculated for each primary outcome to indicate the strength of residual confounding $[9,10]$. The association between missingness in BMI (17.4\%), parity $(0.5 \%)$, and gravida $(0.4 \%)$ with $\mathrm{CP}, \mathrm{LB}$, and miscarriage were assessed using chi-square tests.

\section{Results}

The study cohort consisted of 6795 cycles that met the inclusion and exclusion criteria. Among these, 1138 
cycles underwent blastocyst stage transfer and $5657 \mathrm{cy}-$ cles underwent cleavage stage transfer. The patient baseline characteristics are presented in Table 1 . The overall mean age of patients was $33.5(\mathrm{SD}=3.3)$, and the majority of these women were non-Hispanic White (41\%). Common infertility diagnoses were male infertility (36.0\%), diminished ovarian reserve (28.5\%), and unexplained infertility (15.9\%). The median number of available oocytes was $5[\mathrm{IQR}=3,8]$, and the median number of fertilized oocytes was 2 [IQR $=2,3]$. There were very few excess embryos cryopreserved, $89 \%$ of the women had no excess embryos.

The pregnancy outcomes are as follows. The $\mathrm{CP}$ rate in this study cohort was $39 \%(n=2642)$. Among cycles with CP, the miscarriage rate was $15 \%(n=403)$. The LB rate for the study cohort was 33\% $(n=2215)$. Lastly, among cycles with heart beats, rate of singleton was $83 \%$ $(n=2049 / 2471)$ and rate of twins was $16 \%(n=401 /$
2471). Among single cleavage stage embryo transfer, singleton rate was $99 \%(n=487 / 493)$ and twin rate was $1 \%(n=5 / 493)$. Among multiple cleavage stage embryo transfer, singleton rate was $74 \%(n=1119 / 1522)$ and twin rate was $25 \%(n=383 / 1522)$. Among blastocyst embryos, singleton rate was $97 \%(n=443 / 456)$ and twin rate was $3 \%(n=13 / 456)$. Of note, $81.5 \%(n=2015 / 2471)$ of cycles were cleavage stage transfers.

The regression results are presented in Tables 2 and 3 . Since no statistically significant association between missingness in covariates (BMI, parity, and gravida) and the primary outcomes were observed, complete case analysis was performed. We observed that single blastocyst transfer was associated with an increased odds of clinical pregnancy $(\mathrm{OR} 2.03 ; 95 \% \mathrm{CI}=1.72,2.41 ; E$ value $=2.20)$ and live birth $(\mathrm{OR} 1.86 ; 95 \% \mathrm{CI}=1.55,2.22$; $E$ value $=2.07)$ when compared to single cleavage transfer. No statistically significant association was observed

Table 1 Patient demographics and characteristics

\begin{tabular}{|c|c|c|c|c|c|}
\hline \multirow[t]{2}{*}{ Characteristics } & \multirow{2}{*}{$\begin{array}{l}\text { Single blastocyst } \\
(n=1138)\end{array}$} & \multicolumn{2}{|l|}{ Single cleavage } & \multicolumn{2}{|l|}{$2+$ cleavage } \\
\hline & & $(n=1925)$ & $P$ value ${ }^{a}$ & $(n=3732)$ & $P$ value ${ }^{a}$ \\
\hline Patient's age, mean (SD) & $33(3.4)$ & $33.6(3.2)$ & $<0.0001$ & $33.7(3.3)$ & $<0.0001$ \\
\hline Race/ethnicity, n (\%) & & & 0.08 & & 0.03 \\
\hline Non-Hispanic White & $52(4.6 \%)$ & $138(7.2 \%)$ & & $234(6.3 \%)$ & \\
\hline Non-Hispanic Black & $74(6.5 \%)$ & $126(6.5 \%)$ & & $196(5.3 \%)$ & \\
\hline Other (Asian/American Indian) & $456(40.1 \%)$ & $742(38.5 \%)$ & & $1586(42.5 \%)$ & \\
\hline Hispanic/Latino & $118(10.4 \%)$ & $200(10.4 \%)$ & & $333(8.9 \%)$ & \\
\hline Unknown & $438(38.5 \%)$ & $719(37.4 \%)$ & & $1383(37.1 \%)$ & \\
\hline Recipient's BMI, mean (SD) & $26(5.9)$ & $26.1(6.1)$ & 0.75 & $26.4(6.1)$ & 0.09 \\
\hline Parity, $n$ (\%) & & & 0.09 & & 0.69 \\
\hline Multiparous & $197(17.4 \%)$ & $383(20.0 \%)$ & & $668(18.0 \%)$ & \\
\hline Nulliparous & $935(82.6 \%)$ & $1532(80.0 \%)$ & & $3046(82.0 \%)$ & \\
\hline Gravidity, median [Q1, Q3] & $0[0,1]$ & $0[0,1]$ & 0.31 & $0[0,1]$ & 0.80 \\
\hline Smoker & $45(4.0 \%)$ & $81(4.2 \%)$ & 0.80 & $155(4.2 \%)$ & 0.83 \\
\hline \multicolumn{6}{|l|}{ Infertility diagnoses, $n$ (\%) } \\
\hline Male infertility & $405(35.6 \%)$ & $673(35.0 \%)$ & 0.75 & $1,371(36.7 \%)$ & 0.50 \\
\hline Endometriosis & $140(12.3 \%)$ & $198(10.3 \%)$ & 0.10 & $400(10.7 \%)$ & 0.15 \\
\hline Polycystic ovarian syndrome & $135(11.9 \%)$ & $208(10.8 \%)$ & 0.40 & $426(11.4 \%)$ & 0.72 \\
\hline Diminished ovarian reserve & $279(24.5 \%)$ & $602(31.3 \%)$ & $<0.0001$ & $1057(28.3 \%)$ & 0.01 \\
\hline Tubal ligation/hydrosalpinx/other & $176(15.5 \%)$ & $297(15.4 \%)$ & 1.00 & $584(15.6 \%)$ & 0.92 \\
\hline Uterine factor infertility & $62(5.4 \%)$ & $86(4.5 \%)$ & 0.26 & $150(4.0 \%)$ & 0.05 \\
\hline Unexplained infertility & $174(15.3 \%)$ & $286(14.9 \%)$ & 0.79 & $618(16.6 \%)$ & 0.33 \\
\hline Other infertility diagnosis & $122(10.7 \%)$ & $174(9.0 \%)$ & 0.14 & $248(6.6 \%)$ & $<0.0001$ \\
\hline FSH dose IU, mean (SD) & $3601.9(1776.8)$ & $3622.6(1876.3)$ & 0.77 & $3592.6(1728.9)$ & 0.88 \\
\hline No. of available oocytes, median [Q1, Q3] & $6[4,9]$ & $4[2,7]$ & $<0.0001$ & $6[4,8]$ & 0.84 \\
\hline No. of excess embryos cryopreserved, median [Q1, Q3] & $0[0,1]$ & $0[0,0]$ & $<0.0001$ & $0[0,0]$ & $<0.0001$ \\
\hline Number of fertilized oocytes, median [Q1, Q3] & $2[1,3]$ & $1[1,2]$ & $<0.0001$ & $3[2,3]$ & $<0.0001$ \\
\hline
\end{tabular}

SD standard deviation, 01 25th percentile, Q3 75th percentile

${ }^{\mathrm{a} C o m p a r e d}$ to single blastocyst 
Table 2 Pregnancy outcomes by single blastocyst and single cleavage

\begin{tabular}{|c|c|c|c|c|}
\hline Pregnancy outcomes & Single blastocyst $(n=1138)$ & Single cleavage $(n=1925)$ & OR $(95 \% \mathrm{Cl})$ & $P$ value \\
\hline Clinical pregnancy rate & $489(43 \%)$ & $526(27 \%)$ & $2.03(1.72,2.41)^{\mathrm{a}}$ & $<0.0001$ \\
\hline Live birth rate & $398(35 \%)$ & $439(23 \%)$ & $1.86(1.55,2.22)^{\mathrm{a}}$ & $<0.0001$ \\
\hline Miscarriage rate & $84(17 \%)$ & $84(16 \%)$ & $1.10(0.76,1.59)^{\mathrm{a}}$ & 0.60 \\
\hline Total number of cycles with heart beats & 456 & 493 & - & \\
\hline Singleton rate & $443(97 \%)$ & 487 (99\%) & $0.42(0.16,1.11)^{b}$ & 0.08 \\
\hline Twins rate & $13(3 \%)$ & $5(1 \%)$ & $2.86(1.01,8.10)^{b}$ & 0.05 \\
\hline Triplets & 0 & 1 & - & \\
\hline Quadruplets & 0 & 0 & - & \\
\hline
\end{tabular}

${ }^{a}$ Adjusted for age, race, BMI, smoker, parity, gravida, infertility diagnoses, and assisted hatching

bunadjusted

when comparing single blastocyst transfer to multiple cleavage embryo transfer for clinical pregnancy (OR 0.94; $95 \% \mathrm{CI}=0.81,1.09)$ and live birth $(\mathrm{OR}=0.88 ; 95 \%$ $\mathrm{CI}=0.76,1.03)$. In addition, the association between day of transfer and miscarriage between single blastocyst transfer and single cleavage embryo transfer or between single blastocyst transfer and multiple cleavage embryo transfer was not statistically significant $(\mathrm{OR}=1.10$; $95 \%$ $\mathrm{CI}=0.76,1.59$, and $\mathrm{OR}=1.35 ; 95 \% \mathrm{CI}=0.99,1.83$ ), respectively.

In the unadjusted regression model, the odds of having a singleton among single blastocyst transfer was not significantly different than that among single cleavage stage transfer (OR 0.42; 95\% CI 0.16, 1.11) but was significantly higher compared to multiple cleavage stage transfer (OR 12.27; 95\% CI 6.99, 21.55). On the other hand, the odds of having twins among single blastocyst transfer was significantly higher than that among single cleavage stage transfer (OR 2.86; 95\% CI $=1.01,8.10$ ) and significantly lower than that among multiple cleavage stage transfer (OR 0.09; 95\% CI 0.05, 0.15).

\section{Discussion}

In patients with less than four fertilized embryos, it is difficult to know whether extending culture to blastocyst will improve the patient's outcome. In our respective cohort study, we found that pregnancy outcomes (CP, LB) are significantly improved with single blastocyst transfer compared to single cleavage embryo transfer and similar compared to multiple cleavage transfer in patients with $<4$ fertilized oocytes. We also found that the twin rate is significantly lower with a single blastocyst transfer compared to multiple cleavage stage transfer, decreasing pregnancy complications. Even though blastocyst transfer was not associated with improved outcomes with respect to multiple cleavage embryo transfer, the benefit of blastocyst transfer lies in a reduced multiple rate. In a previous study of our center analyzing SART data, multiple pregnancy rate in patients age $35-37$ was $40.5 \%$ with a 2 blastocyst transfer versus $1.7 \%$ with a single blastocyst transfer and in patients $38-40$ was $34 \%$ with a 2 blastocyst transfer versus $2.0 \%$ with a single blastocyst transfer [11].

Table 3 Pregnancy outcomes by single blastocyst and 2+ cleavage

\begin{tabular}{|c|c|c|c|c|}
\hline Pregnancy outcomes & Single blastocyst $(n=1138)$ & $2+$ cleavage $(n=3732)$ & OR $(95 \% \mathrm{Cl})$ & $P$ value \\
\hline Clinical pregnancy rate & 489 (43\%) & $1627(44 \%)$ & $0.94(0.81,1.09)^{a}$ & 0.43 \\
\hline Live birth rate & $398(35 \%)$ & $1378(37 \%)$ & $0.88(0.76,1.03)^{a}$ & 0.12 \\
\hline Miscarriage rate & $84(17 \%)$ & $235(14 \%)$ & $1.35(0.99,1.83)^{\mathrm{a}}$ & 0.06 \\
\hline Number of embryos transferred, median [Q1, Q3] & - & $2[2,2]$ & - & \\
\hline Total number of cycles with heart beats & 456 & 1,522 & - & \\
\hline Singleton rate & $443(97 \%)$ & $1119(74 \%)$ & $12.27(6.99,21.55)^{b}$ & $<0.0001$ \\
\hline Twins rate & $13(3 \%)$ & $383(25 \%)$ & $0.09(0.05,0.15)^{b}$ & $<0.0001$ \\
\hline Triplets & 0 & 19 & - & \\
\hline Quadruplets & 0 & 1 & - & \\
\hline
\end{tabular}

Q1 25th percentile, Q3 75th percentile

adjusted for age, race, BMI, smoker, parity, gravida, infertility diagnoses, and assisted hatching

bunadjusted 
This question of whether a patient with a low number of fertilized oocytes will have a blastocyst to transfer that leads to a live birth is clinically applicable. However, there are very few studies which address this question. A recent study found higher $\mathrm{CP}$ and $\mathrm{LB}$ rates with cleavage stage transfer versus blastocyst transfer when only one embryo was available for transfer [12]. Instead, many researchers have investigated blastocyst formation and pregnancy outcomes in order to predict which embryo is most capable of producing a pregnancy [1]. Time-lapse morphology has been used to predict which cleavage stage embryos will form blastocysts; however, this technology is often cost prohibitive [13, 14]. Metabolomics and proteomics are currently being investigated to help predict the selection of the optimal embryo, but it is not ready for clinical use [1].

In patients with low numbers of embryos to choose from, the concern is the lack of an embryo to transfer with extended culture. In a study by Jones et al. of good prognosis patients that desired a blastocyst transfer, $7 \%$ of patients did not have a blastocyst to transfer after going forward with extended culture [15]. In poor prognosis patients or in patients with few numbers of embryos, a canceled cycle due to no embryo to transfer is of concern.

Strengths of our study include that the data was abstracted from a large national database. This is also one of the first studies to evaluate pregnancy outcomes in patients with either a cleavage stage or blastocyst transfer and a low number of fertilized eggs.

Limitations of our study include primarily limitations of our data set. The major limitation of our study is the absence of data on how many cycles did not have a transfer due to the cleavage stage embryos failing to reach the blastocyst stage. We also acknowledge that a majority of the cycles in this data set (83\%) were cleavage stage transfers and not blastocyst transfers. Due to the nature of the SART dataset, there is missing data in the database and there may be errors in data reporting. The data is also from 2014 to 2015, and it is not the most current data available. Our study was a retrospective cohort study in design. A limitation of our data is the difference in the baseline characteristics in our subgroups, such as the younger age in single blastocyst transfers versus cleavage stage transfers, which could be a confounding factor. Another limitation is that with an $E$ value around 2, there must exist a confounder with both the exposure and the outcome with an effect size of at least 2. This confounder or confounders could contribute to the results as listed above.

Another major limitation of our data set is that embryo quality information was not available, especially as this relates to embryo quality on day 3 of the blastocyst transfers. It is possible that the embryos on day 3 that had better morphology were the embryos that underwent extended culture and the embryos that had worse morphology were transferred as a cleavage stage embryo.

It is interesting and important to note that most of the patients in our study did have a cleavage stage transfer rather than a blastocyst transfer (likely due having less than four fertilized eggs). However, the pregnancy outcomes (CP, LB) were still improved in the blastocyst group. This finding does not eliminate the possibility of having no embryos for patients who opted for extended culture. This information could be very important for counseling these patients on their options.

\section{Conclusions}

In this retrospective cohort study, pregnancy outcomes (CP, LB) are significantly improved with single blastocyst transfer compared to single cleavage embryo transfer and similar compared to multiple cleavage embryo transfer in patients with $<4$ fertilized oocytes. Multiple pregnancy rates are significantly reduced with single blastocyst transfer compared to multiple cleavage transfer. Based on these findings, IVF programs and patients should consider pursuing extended culture with single blastocyst transfer if more than one embryo with good morphology is available on day 3 .

\section{Abbreviations \\ CP: Clinical pregnancy; IVF: In vitro fertilization; LB: Live birth; SART: Society for Assisted Reproductive Technology}

\section{Acknowledgements \\ The authors thank the Society for Assisted Reproductive Technology as well as all of its members who have submitted data and the patients who have allowed their data to be reported.}

\section{Authors' contributions}

SS, KA, and SM designed the study. TT and CP conducted the statistical analysis. All authors contributed to the writing and revising of the manuscript and approved the final version.

\section{Funding \\ There is no funding for this study, and the authors have no competing interests.}

Availability of data and materials

Data for our study can be found in the SART database.

Ethics approval and consent to participate

Approval was granted by the Duke institutional review board for this retrospective cohort study (Pro00089887). No consent was necessary due to the deidentified nature of our data.

\section{Consent for publication}

Not applicable.

\section{Competing interests}

The authors declare that there are no conflicts of interest.

\section{Author details}

${ }^{1}$ Duke Fertility Center, Duke University, 5704 Fayetteville Road, Durham, NC 27713, USA. ${ }^{2}$ Department of Biostatistics and Bioinformatics, Duke University, 2424 Erwin Road Ste 1105, 11034 Hock Plaza, Durham, NC 27705, USA. 
Received: 14 November 2019 Accepted: 20 January 2020

Published online: 30 January 2020

References

1. Practice Committee of the American Society for Reproductive Medicine, Medicine Practice Committee of the Society for Assisted Reproductive Technology (2018) Blastocyst culture and transfer in clinically assisted reproduction: a committee opinion. Fertil Steril 110:1246-1252

2. Glujovsky D (2016) Cleavage stage versus blastocyst stage embryo transfer in assisted reproductive technology. Cochrane Database Syst Rev. https:// doi.org/10.1002/14651858.CD002118.pub5

3. Papanikolaou EG, Camus M, Kolibianakis EM, Van Landuyt L, Van Steirteghem A, Devroey P (2006) In vitro fertilization with single blastocyststage versus single cleavage-stage embryos. N Engl J Med 354:1139-1146

4. Phillips SJ, Dean NL, Buckett WM, Tan SL (2003) Consecutive transfer of day 3 embryos and of day 5-6 blastocysts increases overall pregnancy rates associated with blastocyst culture. J Assist Reprod Genet 20:461-464

5. Papanikolaou EG, Kolibianakis EM, Tournaye $H$, Venetis CA, Fatemi $H$, Tarlatzis B et al (2008) Live birth rates after transfer of equal number of blastocysts or cleavage-stage embryos in IVF. A systematic review and meta-analysis. Hum Reprod 23:91-99

6. Levitas E, Lunenfeld E, Har-Vardi I, Albotiano S, Sonin Y, Hackmon-Ram R et al (2004) Blastocyst-stage embryo transfer in patients who failed to conceive in three or more day 2-3 embryo transfer cycles: a prospective, randomized study. Fertil Steril 81:567-571

7. Zegers-Hochschild F, Adamson GD, Dyer S, Racowsky C, de Mouzon J, Sokol R, et al (2017) The international glossary on infertility and fertility care Hum Reprod 32:1786-801

8. Kirkegaard K, Kesmodel US, Hindkjaer JJ, Ingerslev HJ (2013) Time-lapse parameters as predictors of blastocyst development and pregnancy outcome in embryos from good prognosis patients: a prospective cohort study. Hum Reprod 28:2643-2651

9. R Development Core Team. R: a language and environment for statistical computing. Version 3.4.4 [software]. 2018 [cited 2019 Jan 18]. Available from: https://www.R-project.org/

10. Mathur MB, Ding P, Riddell CA, VanderWeele TJ (2018) Website and R package for computing E-values. Epidemiology 29(5):e45-e47

11. VanderWeele TJ, Ding P (2017) Sensitivity analysis in observational research: introducing the E-value. Ann Intern Med 167:268-274

12. Keyhan S, Acharya KS, Acharya CR, Yeh JS, Provost MP, Goldfarb JM et al (2016) How compliant are in vitro fertilization member clinics in following embryo transfer guidelines? An analysis of 59,689 fresh first in vitro fertilization autologous cycles from 2011 to 2012. Fertil Steril 106:645-652

13. Xiao JS, Healey M, Talmor A, Vollenhoven B (2019) When only one embryo is available is it better to transfer to day 3 or grow on? Reprod BioMed Online 39:916-923

14. Milewski R, Kuc P, Kuczynska A, Stankiewicz B, Lukaszuk K, Kuczynski W (2015) A predictive model for blastocyst formation based on morphokinetic parameters in time-lapse monitoring of embryo development. J Assist Reprod Genet 32:571-579

15. Jones GM, Trounson AO, Lolatgis N, Wood C (1998) Factors affecting the success of human blastocyst development and pregnancy following in vitro fertilization and embryo transfer. Fertil Steril 70:1022-1029

\section{Publisher's Note}

Springer Nature remains neutral with regard to jurisdictional claims in published maps and institutional affiliations.

\section{Submit your manuscript to a SpringerOpen ${ }^{\circ}$ journal and benefit from:}

- Convenient online submission

- Rigorous peer review

- Open access: articles freely available online

High visibility within the field

- Retaining the copyright to your article

Submit your next manuscript at $\boldsymbol{\nabla}$ springeropen.com 